\title{
Effect of Dynamic Stereoscopic Vision on Balance with Induced Anisometropia
}

\author{
Taeho Jun ${ }^{1}$, Joong-Hwi Kim² \\ ${ }^{1}$ Department of Physical Therapy, Graduate School, Daegu Catholic University \\ 13-13, Hayang-ro, Hayang-eup, Gyeongsan-si, Gyeongsangbuk-do, Republic of Korea \\ ${ }^{2}$ Department of Physical Therapy, Daegu Catholic University \\ 13-13, Hayang-ro, Hayang-eup, Gyeongsan-si, Gyeongsangbuk-do, Republic of Korea
}

Received: December 23, 2020. Revised: June 6, 2021. Accepted: July 5, 2021. Published: July 14, 2021.

\begin{abstract}
The purpose of this study was to investigate the correlation between dynamic stereoscopic vision and balance, and to compare our results to previous studies that sought to determine the correlation between changes in visual information and balance. Thirty-three healthy adults participated in the measurement of balance by inducing anisometropia with $+0.0 \mathrm{D},+2.0 \mathrm{D}$, and $+5.0 \mathrm{D}$ refractive lenses placed in specially made glasses that removed peripheral vision. COP data was collected via force plate and was evaluated by converting it to COP velocity. An $\alpha$ $<0.05$ was considered statistically significant.

Our results show that, provided it is above a minimum threshold level, the greater the change in dynamic stereoscopic vision, the poorer the balance. And it was found that changes below a minimum threshold level did not affect the balance. These results provide a potential explanation for the complementary compensatory action of sensory information, which could not be explained previously based on the change of balance due to changes in visual information across both eyes. Given these data, it seems clear that vision correction is essential for those affected by dynamic stereoscopic vision formation disorders above a certain level in order to improve balance. On the other hand, dynamic stereoscopic vision formation disorders below a certain level can likely be improved using simple somatosensory treatments. This study will help physical therapists better consider dynamic stereoscopic vision when evaluating and conducting therapies designed to increase balance.
\end{abstract}

Keywords - Balance, anisometropia, dynamic stereoscopic vision

\section{INTRODUCTION}

O alance is the maintenance of the center of mass (COM) in the base of support (BOS) and is divided into dynamic and static balance. In order to evaluate functional movement, we focused on dynamic balance [1]-[3].

Balance is required to perform most activities safely, especially activities like walking, for which dynamic balance is required. The one-leg standing balance assessment is commonly used to assess dynamic balance in individuals ranging from athletes with high levels of movement, to elderly patients with increased fall risks [4]-[7].

Balance involves the processing of sensory information and complex motor control of biomechanical factors. Proper balance requires visual information, and vestibular system information and a variety of sensory information including proprioception, cutaneous and joint receptor [8]. Among these, visual input plays perhaps the most vital role in maintaining balance [9].

There are a variety of factors that can affect stereoscopic vision, including anisometropia, accommodative power, retinal size, and illumination. In general, the lower the vision, the lower the stereoscopic vision [10]. Monocular vision is generated by information passing through each individual eye. The information each eye receives is slightly different from the other, allowing the monocular vision to be transformed into 
stereoscopic vision through the process of fusion [11]. Anisometropia interferes with the fusion of the monocular vision, thus preventing the formation of a stereoscopic vision. Stereoscopic vision allows for more accurate recognition of perspective, direction, and speed, as well as affects balance [12]. Stereoscopic vision is divided into static and dynamic, and while some studies have shown that anisometropia does not affect stereoscopic vision, these studies only focused on static stereoscopic vision [13].

Previous studies have shown that to affect dynamic stereoscopic vision, anisometropia requires a difference of 2.0D or more in the refractive power between the two eyes [14],[15]. As balance is a complex control process, it is evaluated by inducing visual changes in normal eyes in order to control for other variables [16].

Herein, we aimed to induce anisometropia while controlling the other variables affecting balance. Additionally, we sought to investigate dynamic balance according to changes in dynamic stereoscopic vision through the one-leg standing balance evaluation.

Table 1. General characteristics of subjects $(\mathrm{N}=33)$

\begin{tabular}{lll}
\hline \multicolumn{2}{l}{ Characteristic } & Mean \pm SD \\
\hline \multirow{2}{*}{ subjects } & Age $(\mathrm{yrs})$ & $22.15 \pm 1.65$ \\
\cline { 2 - 3 } & Subjects Height $(\mathrm{cm})$ & $166.2 \pm 8.74$ \\
\cline { 2 - 3 } & Subjects Weight $(\mathrm{kg})$ & $63.27 \pm 12.19$ \\
\hline
\end{tabular}

${ }^{\mathrm{a}}$ Mean $\pm \mathrm{SD}$

\section{METHODS}

The study was conducted with approval from the institutional ethics committee of Daegu Catholic University (CUIRB-2020-0060).

All study subjects agreed to participate voluntarily and were provided a full explanation of the study purpose.

The study involved 40 healthy adults (men:12, women:28), who had more than 1.0 corrected vision, passed the dynamic stereoscopic vision criteria of the titmus-fly stereotest, and were without musculoskeletal or neurological disorders. 7 adults (men:4, women:3) who failed to maintain one-leg standing for 30 seconds were excluded from the study (Table 1).

The study was carried out by wearing specially designed peripheral vision removal glasses for dynamic stereoscopic vision change and combining +0.0D, +2.0D, and +5.0D refractive lenses on the dominant eye as identified by the $\mathrm{O}$ - ring test. Subjects wore these glasses, stood on a force plate (AMTI, Newton, MA, USA), and looked at a point moving at $1.5 \mathrm{~m} / \mathrm{s}$ on a 75 -inch display installed at a height of $110 \mathrm{~cm}$ located $3 \mathrm{~m}$ in front of the force plate while standing on one leg for 30 seconds (Fig 1). These tests focus on dynamic stereoscopic vision [17]-[19].

To perform the one-leg standing test, subjects stood with both arms in a comfortable position, then one leg was lifted at the hip joint by $20^{\circ}$, and the knee joint was bent $45^{\circ}$. Subjects were told to avoid voluntary movements of the head and hands that could affect balance.

The study consisted of six sets considering the refractive power and one-leg standing direction. The order of the 6 sets was randomized with each set repeated three times, resulting in a total of 18 one-leg stands performed. To reduce muscle and eye fatigue, a 10-minute break was provided between each set.

This study used a force plate to collect the center of pressure (COP) data. The data from the force plate was collected using the A/D(DT3002, DataTranslation, Marlboro, MA, USA) card with a $1000 \mathrm{~Hz}$ sampling frequency. First, the analog data collected through the A/D converter was converted into digital data and stored. Then, the collected data was converted to COP velocity according to the following equation. The resulting value was used as a measure of balance [20]-[22].

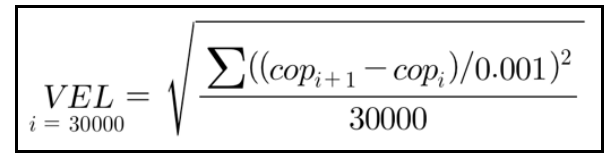

COP velocity is inversely proportional to dynamic balance, and dynamic balance is divided into COPX, COPY, and COPT, based on the $\mathrm{X}, \mathrm{Y}$, and $\mathrm{T}$ axes.

\section{RESULTS}

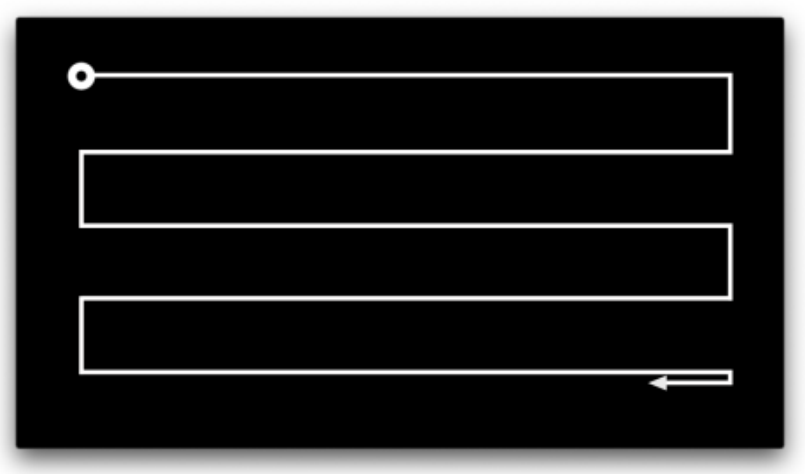

Fig 1. Visual tracking target 
As shown in Fig 2, when the refractive power of the lens inducing anisometropia changed, the balance of the subject also changed (Fig 2). There was a significant difference between $+0.0 \mathrm{D}$ and $+5.0 \mathrm{D}$ induced anisometropia in COPX, COPY, and $\operatorname{COPT}(\mathrm{p}<0.05)$. There were no differences between $+0.0 \mathrm{D}$ and $+2.0 \mathrm{D}$, or $+2.0 \mathrm{D}$ and $+5.0 \mathrm{D}$ induced anisometropia $(\mathrm{p}>0.05)$ (Table 2)(Fig 3).

\section{DISCUSSION}

Visual information is well-known to play an important role in balance and many studies have been conducted on the changes in balance caused by blurred vision [23]. Additionally, studies have been conducted investigating the changes in balance control due to changes in visual information through visual feedback devices [24]. However, an important part of balance in our daily lives is the ability to accurately recognize space and control the body in that space using dynamic stereoscopic vision by getting information about the surroundings while walking or recognizing moving objects when standing. So, in order to study the effect of visual information on balance, a study on the changes in balance caused by anisometropia, which causes a change in dynamic stereoscopic vision, was conducted. Previous studies have identified a correlation between changes in visual information and balance, but did so without regard to dynamic stereoscopic vision. These previous studies concluded that balance is diminished with a severity correlated to the change in visual information. However, in the current study in which we made changes to dynamic stereoscopic vision, we found that changes below a certain threshold level did not

affect balance at all. In short, if the change in dynamic stereoscopic vision is below a certain level, it does not affect the ability to balance, however, if the change in dynamic stereoscopic vision is above a certain level, it will affect the

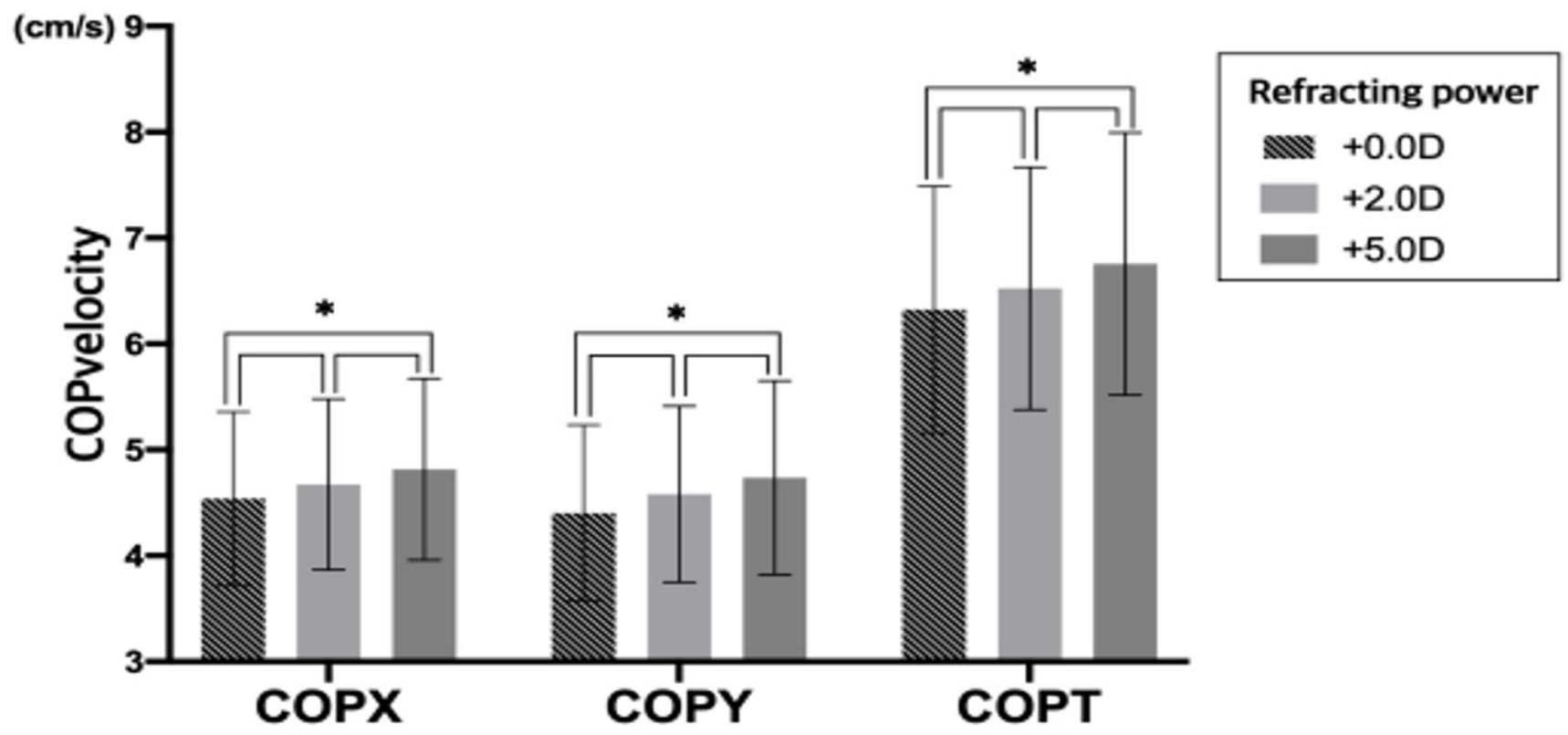

Fig 3. Pairwise comparisons of refracting power for COP velocity graph (COPX, COPY, COPT)

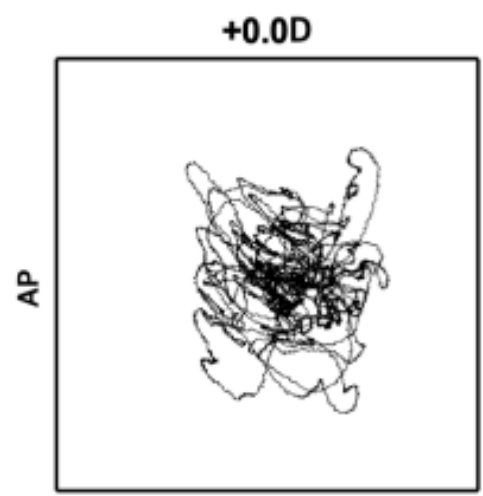

ML

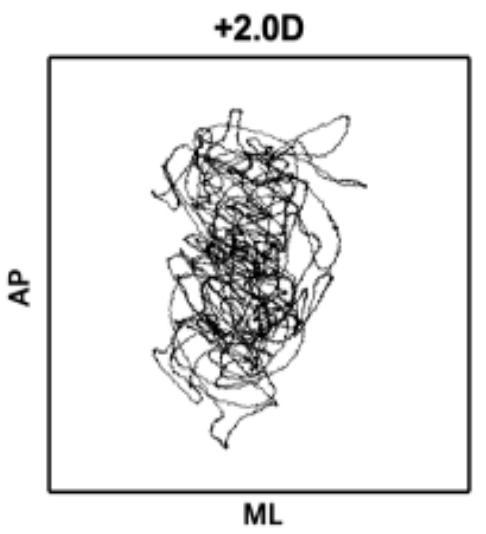

ML

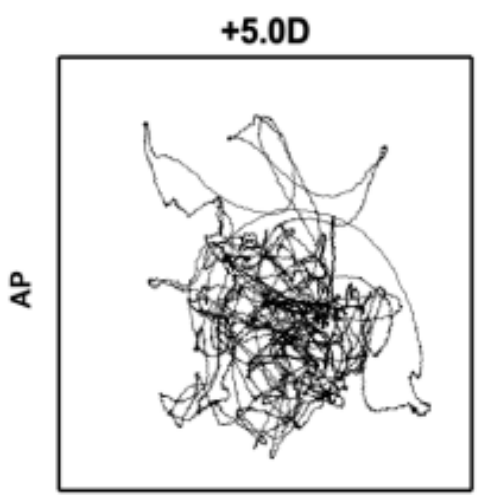

ML

Fig 2. comparisons of refracting power for COP velocity $(\mathrm{N}=12)$ 
Table 2. Pairwise comparisons of refracting power for COP velocity (COPT)

\begin{tabular}{|c|c|c|c|c|c|}
\hline \multirow[t]{2}{*}{ I } & \multirow[t]{2}{*}{$\mathrm{J}$} & \multirow{2}{*}{$\begin{array}{l}\text { Mean Difference } \\
(\mathrm{I}-\mathrm{J})\end{array}$} & \multirow[t]{2}{*}{$\mathrm{p}$} & $\begin{array}{l}95 \% \text { Confidence } \\
\text { Difference }\end{array}$ & Interval for \\
\hline & & & & Lower Bound & Upper Bound \\
\hline \multirow{2}{*}{$+0.0 \mathrm{D}$} & $+2.0 \mathrm{D}$ & -0.199 & 0.250 & -0.491 & 0.094 \\
\hline & $+5.0 \mathrm{D}$ & -0.432 & $0.001 *$ & -0.725 & -0.140 \\
\hline \multirow{2}{*}{$+2.0 \mathrm{D}$} & $+0.0 \mathrm{D}$ & 0.199 & 0.250 & -0.093 & 0.491 \\
\hline & $+5.0 \mathrm{D}$ & -0.234 & 0.146 & -0.527 & 0.058 \\
\hline \multirow{2}{*}{$+5.0 \mathrm{D}$} & $+0.0 \mathrm{D}$ & 0.432 & $0.001 *$ & 0.140 & 0.725 \\
\hline & $+2.0 \mathrm{D}$ & 0.234 & 0.146 & -0.058 & 0.527 \\
\hline
\end{tabular}

$* \mathrm{p}<0.05$

subject's balance.

Balance involves the processing of sensory information through neural systems and subsequent complex motor control of biomechanical factors. Processing sensory information involves visual, vestibular system information and the somatosensory systems including proprioception, cutaneous, and joint receptor [8]. Together, the input of somatosensory information, visual information, and vestibular system information works in combination to form a reliable and precise orientation of the senses, each detecting the position of the body in space. They also play a complementary role in the accurate interpretation of sensory information for orientation and the ability to select each sensory information for balance [8]. Therefore, people who lose vestibular function have to compensate by using visual and somatosensory information in order to properly balance and orient themselves [25]. As such, balance can be adjusted by altering the complementary sensory information, even in the case of information loss or abnormality.

Previously, studies of changes in visual information applied to both eyes simultaneously could not explain the complementary compensation between balance and other sensory information. By identifying changes in balance due to changes in dynamic stereoscopic vision, this study found that the complementary compensation between balance and sensory information could be explained.

Therefore, given these data, vision correction is clearly essential for patients who are at risk of falling or have low balance due to a dynamic stereoscopic formation disorder above a certain threshold. On the other hand, patients with dynamic stereoscopic formation disorders below a certain level can increase their balance through physical therapy or training to enhance their somatosensory system.

These results also suggest that identifying dynamic stereoscopic formation factors when evaluating and treating balance disorders would likely lead to improved clinical outcomes.

\section{CONCLUSION}

These data show that dynamic stereoscopic changes below a certain level do not affect balance, and that dynamic stereoscopic changes above a certain level do affect balance. These results also describe the complementary compensation of somatosensory, vestibular, and visual information in balance. In short, this study found that dynamic stereoscopic formation disorders do affect balance when above a certain threshold, and that stereoscopic formation disorders below a certain level do not affect balance due to the compensation of somatosensory, vestibular, and visual systems. And this study found that this effect occurs on the 5D difference criterion for refractive power. However, it is difficult to determine the accurate prove of refractive power threshold level for complementary compensation of balance. Therefore, more follow-up studies are needed to generalize and scientifically accurate prove the refractive power for complementary compensation of balance. Nevertheless, this study showed that physical therapists should consider dynamic stereoscopic vision during the evaluation and treatment of balance disorders.

\section{ACKNOWLEDGMENTS}

This work was supported by the sabbatical research grant from Daegu Catholic University in 2019.

\section{References}

[1] D. A. Winter, "Human balance and posture control during standing and walking," Gait \& posture, Vol. 3, No.4, pp. 193-214, 1995.

[2] P. J. Stapley, T. Pozzo, "Does the coordination between posture and movement during human whole-body reaching ensure center of mass stabilization?," Experimental brain research, Vol. 129, No. 1 (1999): 134-146. 
[3] T. C. Sell, "An examination, correlation, and comparison of static and dynamic measures of postural stability in healthy, physically active adults," Physical therapy in sport : official journal of the Association of Chartered Physiotherapists in Sports Medicine, Vol. 13, No. 2, pp.80-86, 2012.

[4] A. Promsri, T. Haid, "How does lower limb dominance influence postural control movements during single leg stance?," Human movement science, Vol. 58, pp. 165-174, 2018.

[5] T. Hahn, A. Foldspang, "One-leg standing balance and sports activity," Scandinavian journal of medicine \& science in sports, Vol. 9, No. 1, pp. 15-18, 1999.

[6] S-Y. Chew-Bullock, D. I. Anderson, "Kicking performance in relation to balance ability over the support leg," Human movement science, Vol. 31, No. 6, pp. 1615-1623, 2012.

[7] R. A. da Silva, M. Bilodeau, "Age-related differences in time-limit performance and force platform-based balance measures during one-leg stance," J Electromyogr Kinesiol. Vol. 23, No. 3, pp. 634-649, Jun, 2013.

[8] A. Shumway-Cook, F. B. Horak, "Assessing the influence of sensory interaction of balance. Suggestion from the field," Physical therapy, Vol. 66, No. 10, pp. 1548-1550, 1986.

[9] M. S. Redfern, L. Yardley, "Visual influences on balance," Journal of anxiety disorders, Vol. 15, No. 1-2, pp. 81-94, 2001.

[10] J. V. Lovasik, M. Szymkiw, "Effects of aniseikonia, anisometropia, accommodation, retinal illuminance, and pupil size on stereopsis," Investigative ophthalmology \& visual science, Vol. 26, No. 5, pp. 741-750, 1985.

[11] J. I. Nelson, "Globality and stereoscopic fusion in binocular vision," Journal of theoretical biology, Vol. 49, No. 1, pp. 1-88, 1975.

[12] S. R. Lord, H. B. Menz, "Visual contributions to postural stability in older adults," Gerontology, Vol. 46, No. 6, pp. 306-310, 2000.

[13] L. A. Levin, P. L Kaufman, Adler's physiology of the eye. 11th, ELSEVIER, 2011.

[14]M. Gawęcki, "Threshold Values of Myopic Anisometropia Causing Loss of Stereopsis," Journal of ophthalmology, Vol. 2019, No. 6, pp 1-8, 2019.

[15] C. Zetterlund, L-O. Lundqvist, "Visual, musculoskeletal and balance symptoms in individuals with visual impairment," Clinical \& experimental optometry, Vol. 102, No. 1, pp. 63-69, 2019.

[16] V. Anand, J. Buckley, "The effect of refractive blur on postural stability," Ophthalmic \& physiological optics : the journal of the British College of Ophthalmic Opticians (Optometrists), Vol. 22, No. 6, pp. 528-534, 2002.

[17] A. S. Edwards, "Body sway and vision," Journal of experimental psychology, Vol. 36, No. 6, pp. 526-535, 1946.

[18] Bianchi, Luigi. "Speedy'O’Brain: a neuro-feedback videogame driven by electroencephalographic signals." International Journal of Biology and Biomedical Engineering, Vol. 12, pp. 229-234, 2018.

[19] Lai, Ying-Fang, Chien-Yuan Lai, and Hsiu-Sen

Chiang. "Application of music listening and EEG analysis for sustained attention

training." International Journal of Biology and Biomedical Engineering. Vol. 9, pp. 133-140, 2015.

[20] P. A. Gribble, J. Hertel, "Effect of hip and ankle muscle fatigue on unipedal postural control," Journal of electromyography and kinesiology : official journal of the International Society of Electrophysiological Kinesiology, Vol. 14, No. 6, pp. 641-646, 2004.

[21] A. C. Geurts, B. Nienhuis, "Intrasubject variability of selected force-platform parameters in the quantification of postural control," Archives of physical medicine and rehabilitation, Vol. 74, No. 11, pp. 1144-1150, 1993.

[22] Jung, Ji-Yong, Chang-Min Yang, Jung-Ja Kim. "Gait analysis for elderly people with visual impairment using plantar pressure measurement." ." International Journal of Biology and Biomedical Engineering. Vol. 11, pp. 187-193, 2017.

[23] S. R. Lord, J. Dayhew, "Visual risk factors for falls in older people," Age and ageing, Vol. 35, No. 2, pp. ii42-ii45, 2006.

[24] R. G. Hamman, I. Mekjavic, "Training effects during repeated therapy sessions of balance training using visual feedback," Archives of physical medicine and rehabilitation, Vol. 73, No. 8, pp. 738-744, 1992.

[25]F. B. Horak, L. M. Nashner, "Postural strategies associated with somatosensory and vestibular loss," Experimental brain research, Vol. 82, No. 1, pp. 167-177, 1990. 
Contribution of individual authors to the creation of a scientific article (ghostwriting policy)

Author's Contributions.

All authors contributed to this study:

Taeho Jun - original draft preparation, conceptualization, data curation, data analysis

joong-Hwi Kim - methodology, data analysis, supervision

Creative Commons Attribution License 4.0 (Attribution 4.0 International, CC BY 4.0)

This article is published under the terms of the Creative Commons Attribution License 4.0

https://creativecommons.org/licenses/by/4.0/deed.en US 